\title{
A NOTE ON RUIN PROBLEMS IN PERTURBED CLASSICAL RISK MODELS
}

\author{
PENG LIU, CHUNSHENG ZHANG, AND LANPENG JI
}

\begin{abstract}
In this short note, we derive explicit formulas for the joint densities of the time to ruin and the number of claims until ruin in perturbed classical risk models, by constructing several auxiliary random processes.
\end{abstract}

\section{INTRODUCTION}

Consider the surplus process $\left\{U_{u}(t), t \geq 0\right\}$ of an insurance company modeled by

$$
U_{u}(t)=u+c t-\sum_{i=1}^{N(t)} X_{i}+\sigma B(t), \quad t \geq 0,
$$

where $u \geq 0$ is the initial reserve, $c>0$ is the rate of premium income, $\{N(t), t \geq 0\}$ is a Poisson claim-counting process with parameter $\lambda>0,\left\{X_{i}, i \geq 1\right\}$ is a sequence of independent and identically distributed (iid) positive random variables with common survival function $\bar{P}(x)$ and density function $p(x)$, representing the amounts of the successive claims, $\{B(t), t \geq 0\}$ is a standard Brownian motion and $\sigma>0$ is a constant representing the diffusion volatility parameter. In addition, we assume that $\{N(t), t \geq 0\},\{B(t), t \geq 0\}$ and $\left\{X_{i}, i \geq 1\right\}$ are mutually independent, and further suppose that the positive net profit condition $c>\lambda \mathbb{E}\left(X_{1}\right)$ holds. In the literature the risk model (1) is refereed to as the (diffusion) perturbed classical risk model.

Since the introduction of the perturbed classical risk model in the seminal contributions Gerber (1970) and Dufresne and Gerber (1991), the study of it has become popular in risk theory; see, e.g., Zhang and Wang (2003), Li (2006) and Tsai and Lu (2010). We refer to Asmussen and Albrecher (2010) for a nice review on this model and its generalizations.

Define

$$
T_{u}=\inf \left\{t \geq 0: U_{u}(t)<0\right\} \quad(\text { with } \inf \{\emptyset\}=\infty)
$$

to be the time to ruin of the risk model (1) with initial reserve $u \geq 0$. Then $N\left(T_{u}\right)$ is the number of claims until ruin (including the claim which causes ruin). In this paper, we are interested in the calculation of the joint density of $N\left(T_{u}\right)$ and $T_{u}$ defined by

$$
\omega_{u}(n, t)=\frac{\partial}{\partial t} \psi_{u}(n, t), \quad n \in \mathbb{N}_{0}:=\mathbb{N} \cup\{0\}, t>0
$$

Date: September 16, 2016.

Key words and phrases. Perturbed classical risk model, joint density, time to ruin, number of claims until ruin, the Lundberg fundamental equation, martingale. 
with

$$
\psi_{u}(n, t)=\mathbb{P}\left(N\left(T_{u}\right)=n, T_{u} \leq t\right), \quad n \in \mathbb{N}_{0}, t>0 .
$$

A nice formula for $\omega_{u}(n, t)$ in the classical risk model without perturbation (i.e., $\sigma=0$ ) has been discussed in Dickson (2012). For other related results we refer to Egídio dos Reis (2002), Borovkov and Dickson (2008), Landriault et al. (2011), Lee (2011), Frostig et al. (2012), Zhao and Zhang (2013), Cheung (2013), Li et al. (2013), Landriault and Shi (2014) and Li et al. (2016). It is noted that in most of the aforementioned papers results are obtained based on an application of the known Lagrange's Expansion Theorem. For instance, in the framework of Dickson (2012) (see Eq. (3) therein) the derivation of $\omega_{0}(n, t)$ relies heavily on an adequate form of the inverse Laplace transform of the function $\exp \left(-\eta_{\delta} x\right), \delta, x>0$, with respect to $\delta$ (which was found by Lagrange's Expansion Theorem). Here $\eta_{\delta}$ is the unique positive solution of the Lundberg fundamental equation

$$
c s-(\lambda+\delta)+\lambda r \hat{p}(s)=0
$$

with $r \in(0,1], \delta>0$ and $\hat{p}$ the Laplace transform of $p$. We note in passing that the above Lundberg fundamental equation was derived in Landriault et al. (2011) by introducing the following quantity

$$
\phi_{r, \delta}(u)=\mathbb{E}\left(r^{N\left(T_{u}\right)} e^{-\delta T_{u}} I_{\left(T_{u}<\infty\right)}\right)
$$

with $I_{(\cdot)}$ the indicator function.

However, it seems that the approach of Dickson (2012) does not work well anymore for the perturbed risk model (1). One reason is that now $\omega_{0}(n, t)=0$ for all $n \in \mathbb{N}$ by properties of Brownian motion. In this paper, we shall adopt a different approach. Similarly to the unperturbed case, the following generalized Lundberg fundamental equation

$$
f(s)=\frac{\sigma^{2}}{2} s^{2}+c s-(\lambda+\delta)+\lambda r \hat{p}(s)=0
$$

shall play an important role. In fact, the generalized Lundberg fundamental equation was introduced to ensure that, for such $s$ satisfying $f(s)=0$,

$$
\left\{r^{N(t)} e^{s U_{u}(t)-\delta t}, t \geq 0\right\}=\left\{e^{s U_{u}(t)-\delta t+\sum_{i=1}^{N(t)} \ln r}, t \geq 0\right\}
$$

is a martingale with respect to the natural filtration $\left\{\mathcal{F}_{t}^{U_{0}}, t \geq 0\right\}$ generated by $\left\{U_{0}(t), t \geq 0\right\}$. Since, for any fixed $s>0,\left\{s U_{u}(t)-\delta t+\sum_{i=1}^{N(t)} \ln r, t \geq 0\right\}$ is a spectrally negative Lévy process, the above martingale property is equivalent to that

$$
\mathbb{E}\left(e^{s U_{u}(t)-\delta t+\sum_{i=1}^{N(t)} \ln r} \mid U_{u}(0)=u\right)=e^{s u}, \quad \forall t \geq 0,
$$

which is valid if and only if $f(s)=0$. Note that similar argumentations can also be found in Landriault and Shi (2014). Furthermore, since

$$
f(0)<0, \quad \lim _{s \rightarrow \infty} f(s)=\infty, \quad f^{\prime}(s)=\sigma^{2} s+c+\lambda r \hat{p}^{\prime}(s) \geq \sigma^{2} s+c-\lambda r \mathbb{E}\left(X_{1}\right) \geq \sigma^{2} s>0, \forall s>0,
$$


we conclude that the generalized Lundberg fundamental equation $f(s)=0$ has a unique positive solution, denoted by $\rho_{\delta}$. As a result

$$
\left\{e^{\widehat{U}_{u}(t)}, t \geq 0\right\}
$$

with $\widehat{U}_{u}(t):=\rho_{\delta} U_{u}(t)-\delta t+\sum_{i=1}^{N(t)} \ln r, t \geq 0$, is a martingale.

Outline of this paper: In Proposition 1 we derive an adequate expansion for the key function $\exp \left(-\rho_{\delta} x\right)$ by using a probabilistic approach instead of resorting to Lagrange's Expansion Theorem. With the aid of this expansion and the duality of the risk process $\left\{U_{u}(t), t \geq 0\right\}$, we derive (in Theorem 4 ) explicit formulas for the joint density $\omega_{u}(n, t)$ for any $u>0$. In comparison to the classical analytic approach (as in Dickson (2012)) by utilizing Laplace transforms, our probabilistic approach yields much neater formulas. The proofs of all the results are relegated to Section 3.

\section{Results}

Before giving the principle result of this contribution we shall present some preliminary results, among which Proposition 1 shall play a crucial role. We begin with some further notation. For any fixed $x>u$ we define

$$
\tau_{u}^{x}=\inf \left\{t \geq 0: U_{u}(t)=x\right\}
$$

to be the first hitting time to level $x$ of the risk process $\left\{U_{u}(t), t \geq 0\right\}$. Further, define, for any $t>0, x \in \mathbb{R}$

$$
\begin{aligned}
& g_{t}(0, x)=\frac{1}{2 \sqrt{\pi D t}} e^{-\left(\lambda t+\frac{(x-c t)^{2}}{4 D t}\right)} \\
& g_{t}(n, x)=e^{-\lambda t} \frac{(\lambda t)^{n}}{n !} \int_{0}^{\infty} \frac{1}{2 \sqrt{\pi D t}} e^{-\frac{(z+x-c t)^{2}}{4 D t}} p^{n *}(z) d z, \quad n \in \mathbb{N},
\end{aligned}
$$

where $D=\sigma^{2} / 2$ and $p^{n *}, n \in \mathbb{N}$ denotes the $n$-fold convolution of $p$ with itself. It can be shown that $g_{t}(n, x)$ is the joint density of $N(t)$ and $U_{u}(t)-u$, which is independent of the ruin problem considered in the paper.

Proposition 1. Let $\rho_{\delta}$ be the unique positive solution of the generalized Lundberg fundamental equation (4). Then, for any $x>0$

$$
e^{-\rho_{\delta} x}=\sum_{n=0}^{\infty} r^{n} \int_{0}^{\infty} e^{-\delta s} \frac{x}{s} g_{s}(n, x) d s .
$$

Remark 1. The last result was first derived in Landriault and Shi (2014) by using an analytic approach. In comparison to the approach therein, our probabilistic approach results in a much shorter proof.

As an application of Proposition 1 we obtain the following result concerning the joint density of the first hitting time and the number of claims until this hitting time. This result is important for the derivation of Proposition 3 given below. 
Proposition 2. Let $\tau_{u}^{x}$ be the first hitting time to a level $x(>u)$ of the risk process $\left\{U_{u}(t), t \geq 0\right\}$. Then

$$
\mathbb{P}\left(N\left(\tau_{u}^{x}\right)=n, \tau_{u}^{x} \in[t, t+d t]\right)=\frac{x-u}{t} g_{t}(n, x-u) d t, \quad n \in \mathbb{N}_{0}, t>0 .
$$

In order to proceed with the joint density $\omega_{u}(n, t)$ for any $u>0$, we introduce a quantity $H(n, t, u, x), n \in$ $\mathbb{N}_{0}, t, u, x>0$ as follows (recall $T_{u}$ is the time to ruin given in (2))

$$
H(n, t, u, x) d x=\mathbb{P}\left(N(t)=n, t<T_{u}, U_{u}(t) \in[x, x+d x]\right) .
$$

Note that, for any fixed $u>0, H(n, t, u, x), n \in \mathbb{N}_{0}, x>0$ can be interpreted as the joint density of $N(t)$ and $U_{u}(t)$, with $t$ some fixed time before ruin occurs. As it will be seen from our principle result below (Theorem 4 ) $H(n, t, u, x)$ is a crucial quantity for the joint density $\omega_{u}(n, t)$. We present below an explicit expression for it.

Proposition 3. Let $H(n, t, u, x), n \in \mathbb{N}_{0}, t, u, x>0$ be defined above. Then

$$
H(n, t, u, x)=g_{t}(n, x-u)-\sum_{l=0}^{n} \int_{0}^{t} \frac{x}{s} g_{s}(l, x) g_{t-s}(n-l,-u) d s
$$

holds for any $n \in \mathbb{N}_{0}, t, u, x>0$.

Now we are ready to present our principle result concerning the joint density $\omega_{u}(n, t)$ of $N\left(T_{u}\right)$ and $T_{u}$ for any $u>0$. It is known that ruin of the perturbed classical risk model (1) is caused either by a claim or by oscillation; see, e.g., Dufresne and Gerber (1991). In the following denote by $\omega_{u}^{s}(n, t), n \in \mathbb{N}_{0}, t>0$ the joint density when the ruin is caused by a claim, and by $\omega_{u}^{d}(n, t), n \in \mathbb{N}_{0}, t>0$ the joint density when the ruin is caused by oscillation. That is,

$$
\omega_{u}^{s}(n, t)=\frac{\partial}{\partial t} \psi_{u}^{s}(n, t), \quad \omega_{u}^{d}(n, t)=\frac{\partial}{\partial t} \psi_{u}^{d}(n, t), n \in \mathbb{N}_{0}, t>0
$$

with

$$
\begin{aligned}
& \psi_{u}^{s}(n, t)=\mathbb{P}\left(N\left(T_{u}\right)=n, T_{u} \leq t, U_{u}\left(T_{u}\right)<0\right), \\
& \psi_{u}^{d}(n, t)=\mathbb{P}\left(N\left(T_{u}\right)=n, T_{u} \leq t, U_{u}\left(T_{u}\right)=0\right), n \in \mathbb{N}_{0}, t>0 .
\end{aligned}
$$

Clearly, for any $u>0$

$$
\omega_{u}(n, t)=\omega_{u}^{s}(n, t)+\omega_{u}^{d}(n, t), \quad n \in \mathbb{N}_{0}, t>0 .
$$

Our main result below presents explicit expressions for $\omega_{u}^{s}(n, t)$ and $\omega_{u}^{d}(n, t), n \in \mathbb{N}_{0}, t>0$, and thus in view of the above formula yields an explicit expression for the joint density $\omega_{u}(n, t), n \in \mathbb{N}_{0}, t>0$.

Theorem 4. Let $\omega_{u}^{s}(n, t)$ and $\omega_{u}^{d}(n, t), n \in \mathbb{N}_{0}, t>0$ be the joint densities of $N\left(T_{u}\right)$ and $T_{u}$ defined above. Then, for any $u>0$

$$
\omega_{u}^{s}(0, t)=0, \quad \omega_{u}^{d}(0, t)=\frac{u}{2 \sqrt{\pi D t^{3}}} e^{-\left(\lambda t+\frac{(u+c t)^{2}}{4 D t}\right)}, \quad t>0
$$


and

$$
\begin{aligned}
& \omega_{u}^{s}(n, t)=\lambda \int_{0}^{\infty} H(n-1, t, u, x) \bar{P}(x) d x, \quad n \in \mathbb{N}, t>0, \\
& \omega_{u}^{d}(n, t)=\lambda \int_{0}^{t} \int_{0}^{\infty} \int_{0}^{y} H(n-1, t-s, u, y) \frac{y-z}{2 \sqrt{\pi D s^{3}}} e^{-\left(\lambda s+\frac{(y-z+c s)^{2}}{4 D}\right)} p(z) d z d y d s, \quad n \in \mathbb{N}, t>0,
\end{aligned}
$$

where $H(n, t, u, x), n \in \mathbb{N}_{0}, t, u, x>0$ is given in Proposition 3.

\section{Proofs}

In this section we present all the proofs of the results.

Proof of Proposition 1: For any fixed $r \in(0,1]$ define an auxiliary process $\left\{\widetilde{U}_{u}(t), t \geq 0\right\}$ from (1) as follows:

$$
\widetilde{U}_{u}(t)=u+c t-\sum_{i=1}^{N(t)} \widetilde{X}_{i}+\sigma B(t), \quad t \geq 0,
$$

where $\left\{\widetilde{X}_{i}, i \geq 1\right\}$ is a sequence of iid positive random variables such that $\widetilde{X}_{1}$ has density $\widetilde{p}(x)=r p(x), x>0$ and $\mathbb{P}\left(\widetilde{X}_{1}=\infty\right)=1-r$. It is worth remarking at this point that the theorems and corollaries in Chapter VII in Bertoin (1996) still hold for spectrally negative Lévy processes when the domain of the corresponding Lévy measure is generalized from $(-\infty, 0)$ to $(-\infty, 0) \cup\{-\infty\}$. Therefore, by denoting

$$
\widetilde{\tau}_{u}^{x}=\inf \left\{t \geq 0: \widetilde{U}_{u}(t)=x\right\}, \quad x>u,
$$

we have from Corollary 3 in Chapter VII in Bertoin (1996) that the measures $t \mathbb{P}\left(\widetilde{\tau}_{u}^{x} \in[t, t+d t]\right) d x$ and $(x-$ $u) \mathbb{P}\left(\widetilde{U}_{u}(t) \in[x, x+d x]\right) d t$ coincide on $[0, \infty) \times[0, \infty)$. This implies that

$$
\mathbb{P}\left(\widetilde{\tau}_{u}^{x} \in[t, t+d t]\right)=\frac{x-u}{t} \widetilde{g}_{t}(r, x-u) d t,
$$

where

$$
\begin{aligned}
\widetilde{g}_{t}(r, x)= & \frac{\partial}{\partial x} \mathbb{P}\left(\widetilde{U}_{u}(t) \leq x+u\right)=\frac{1}{2 \sqrt{\pi D t}} e^{-\left(\lambda t+\frac{(x-c t)^{2}}{4 D t}\right)} \\
& +\sum_{n=1}^{\infty} e^{-\lambda t} \frac{(\lambda t)^{n}}{n !} \int_{0}^{\infty} \frac{1}{2 \sqrt{\pi D t}} e^{-\frac{(z+x-c t)^{2}}{4 D t}}(r p)^{n *}(z) d z \\
= & \sum_{n=0}^{\infty} r^{n} g_{t}(n, x), \quad x>0 .
\end{aligned}
$$

Moreover, using similar arguments as in Theorem 1 in Chapter VII in Bertoin (1996) we obtain that, for any $\delta>0$

$$
\mathbb{E}\left(e^{-\delta \widetilde{\tau}_{u}^{x}}\right)=\mathbb{E}\left(e^{-\delta \widetilde{\tau}_{u}^{x}} I_{\left(\widetilde{\tau}_{u}^{x}<\infty\right)}\right)=e^{-\rho_{\delta}(x-u)}, \quad x>u .
$$

Consequently, we conclude from (13)-(14) that

$$
e^{-\rho_{\delta}(x-u)}=\sum_{n=0}^{\infty} r^{n} \int_{0}^{\infty} e^{-\delta t} \frac{(x-u)}{t} g_{t}(n, x-u) d t, \quad x>u,
$$



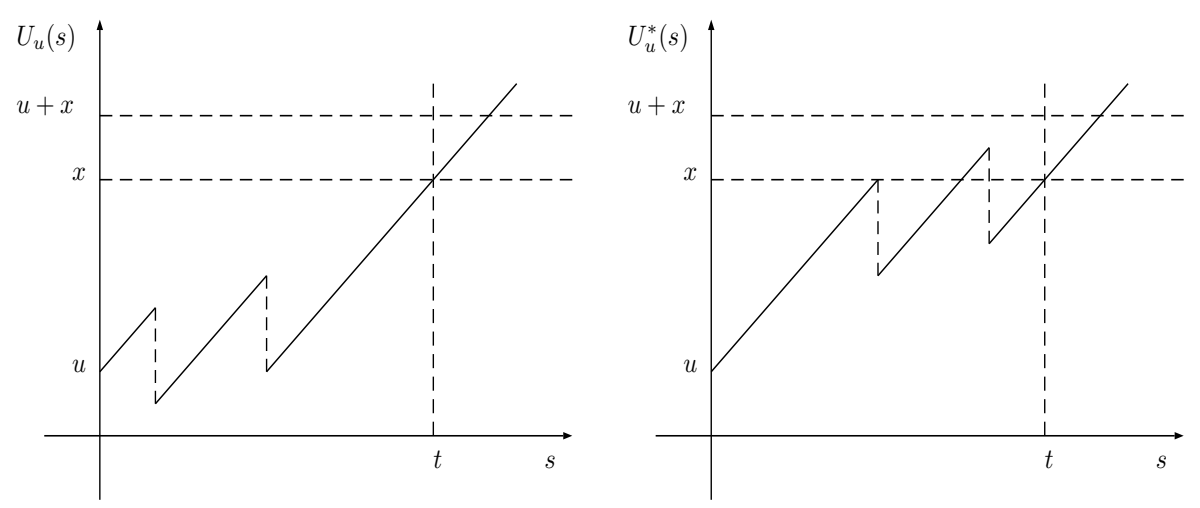

Figure 1. Sample paths of $U_{u}(s)$ and $U_{u}^{*}(s)$ when $\sigma=0$

implying (7). This completes the proof.

Proof of Proposition 2: Recalling (5), we have that, for any fixed $r \in(0,1], \delta>0$,

$$
\left\{e^{\widehat{U}_{u}(t)}, t \geq 0\right\}
$$

is a martingale. Since $\tau_{u}^{x}$ is a stopping time with respect to the filtration $\left\{\mathcal{F}_{t}^{U_{0}}, t \geq 0\right\}$ and

$$
e^{\widehat{U}_{u}\left(t \wedge \tau_{u}^{x}\right)} \leq e^{\rho_{\delta} x}<\infty, \quad \forall t \geq 0,
$$

we have from the optional sampling theorem and the dominated convergence theorem that

$$
\mathbb{E}\left(e^{-\delta \tau_{u}^{x}} r^{N\left(\tau_{u}^{x}\right)} e^{\rho_{\delta} x}\right)=\mathbb{E}\left(e^{\widehat{U}_{u}\left(\tau_{u}^{x}\right)} \mid \widehat{U}_{u}(0)=\rho_{\delta} u\right)=e^{\rho_{\delta} u}
$$

which means

$$
\mathbb{E}\left(e^{\left.-\delta \tau_{u}^{x} r^{N\left(\tau_{u}^{x}\right)}\right)}\right)=e^{-\rho_{\delta}(x-u)} .
$$

Consequently, the claim follows by inserting (7) into the last formula. The proof is complete.

Proof of Proposition 3: We introduce the following quantity:

$$
H^{*}(n, t, u, x) d x=\mathbb{P}\left(N^{*}(t)=n, t<\tau_{* u}^{x+u}, U_{u}^{*}(t) \in[x, x+d x]\right), \quad n \in \mathbb{N}_{0}, t, u, x>0,
$$

where, for the fixed $t>0$,

$$
U_{u}^{*}(s)= \begin{cases}u+U_{u}(t)-U_{u}((t-s)-), & 0 \leq s<t \\ U_{u}(s), & s \geq t\end{cases}
$$

$\tau_{* u}^{x+u}$ is defined as

$$
\tau_{* u}^{x+u}=\inf \left\{s \geq 0: U_{u}^{*}(s)=u+x\right\}, x>0,
$$

and $N^{*}(t)$ is the number of jumps of the process $\left\{U_{u}^{*}(s), s \geq 0\right\}$ until time $t$. See Figure 1 for the sample paths of $U_{u}(s)$ and $U_{u}^{*}(s)$ when $\sigma=0$. It is noted that the construction of $\left\{U_{u}^{*}(s), s \geq 0\right\}$ from $\left\{U_{u}(s), s \geq 0\right\}$ is 
measure-preserved; see, e.g., Gerber and Shiu (1997) and Gerber and Shiu (1998). Therefore, we have

$$
\mathbb{P}\left(N(t)=n, t<T_{u}, U_{u}(t) \in[x, x+d x]\right)=\mathbb{P}\left(N^{*}(t)=n, t<\tau_{* u}^{x+u}, U_{u}^{*}(t) \in[x, x+d x]\right),
$$

which means that

$$
H(n, t, u, x)=H^{*}(n, t, u, x), \quad n \in \mathbb{N}_{0}, t, u, x>0 .
$$

Furthermore, since $\left\{U_{u}^{*}(s), s \geq 0\right\}$ is also a Lévy process and $U_{u}^{*}(t)=U_{u}(t)$, by Theorem 7.10 in Sato (1999) we have the process $\left\{U_{u}^{*}(s), s \geq 0\right\}$ has the same probability law as the process $\left\{U_{u}(s), s \geq 0\right\}$. Thus,

$$
\begin{aligned}
H(n, t, u, x) d x & =H^{*}(n, t, u, x) d x \\
& =\mathbb{P}\left(N(t)=n, t<\tau_{u}^{x+u}, U_{u}(t) \in[x, x+d x]\right) \\
& =\mathbb{P}\left(N(t)=n, U_{u}(t) \in[x, x+d x]\right)-\mathbb{P}\left(N(t)=n, t \geq \tau_{u}^{x+u}, U_{u}(t) \in[x, x+d x]\right)
\end{aligned}
$$

By the homogeneity and strong Markov property of $\left\{U_{u}(s), s \geq 0\right\}$ we conclude that

$$
\begin{aligned}
& \mathbb{P}\left(N(t)=n, t \geq \tau_{u}^{x+u}, U_{u}(t) \in[x, x+d x]\right) \\
& =\sum_{l=0}^{n} \mathbb{E}\left(I_{\left(N\left(\tau_{u}^{x+u}\right)=l, \tau_{u}^{x+u} \leq t\right)} \mathbb{P}\left(N(t)-N\left(\tau_{u}^{x+u}\right)=n-l, U_{u}(t) \in[x, x+d x] \mid \mathcal{F}_{\tau_{u}^{x+u}}^{U_{0}}\right)\right) \\
& =\sum_{l=0}^{n} \int_{0}^{t} \mathbb{P}\left(N(s)=l, \tau_{0}^{x} \in[s, s+d s]\right) \mathbb{P}\left(N(t-s)=n-l, U_{0}(t-s) \in[-u,-u+d x]\right) .
\end{aligned}
$$

Consequently, the claim follows from (6) and (8). This completes the proof.

Proof of Theorem 4: It follows easily that $\omega_{u}^{s}(0, t)=0$. Further, we have

$$
\begin{aligned}
\omega_{u}^{d}(0, t) d t & =\mathbb{P}\left(N(t)=0, \inf _{s \in[0, t)}(u+c s+\sigma B(s))>0, \inf _{s \in[t, t+d t]}(u+c s+\sigma B(s)) \leq 0\right) \\
& =\frac{u}{2 \sqrt{\pi D t^{3}}} e^{-\left(\lambda t+\frac{(u+c t)^{2}}{4 D t}\right)} d t,
\end{aligned}
$$

where we used the formula for the density of hitting time of a drifted Brownian motion, see, e.g., Remark 8.3 in Chapter 2 in Karatzas and Shreve (1988).

Next we consider $\omega_{u}^{s}(n, t), n \in \mathbb{N}, t \geq 0$. It is noted that $\omega_{u}^{s}(n, t) d t$ can be seen as the probability that there are $n-1$ claims until a pre-ruin time $t$ and $U_{u}(t) \in[x, x+d x]$ and there is a claim (which causes ruin) in $[t, t+d t]$. Thus,

$$
\begin{aligned}
\omega_{u}^{s}(n, t) d t & =\int_{0}^{\infty} \int_{0}^{\infty} H(n-1, t, u, x)(\lambda d t) p(x+y) d y d x \\
& =\lambda \int_{0}^{\infty} H(n-1, t, u, x) \bar{P}(x) d x d t .
\end{aligned}
$$

Similarly, $\omega_{u}^{d}(n, t) d t$ can be seen as the probability that there are $n-1$ claims until some pre-last claim occurring time $s$ and there is a claim (which does not cause ruin) in $[s, s+d s]$ and further ruin occurs in $[t, t+d t]$ by 
oscillation. Therefore,

$$
\omega_{u}^{d}(n, t) d t=\int_{0}^{\infty} \int_{0}^{y} \int_{0}^{t} H(n-1, s, u, y) \lambda d s p(z) d z \frac{y-z}{2 \sqrt{\pi D(t-s)^{3}}} e^{-\left(\lambda(t-s)+\frac{(y-z+c(t-s))^{2}}{4 D(t-s)}\right)} d y,
$$

and thus the proof is complete.

Acknowledgements: We are thankful to the editor, the associate editor and two referees for several suggestions which significantly improved our manuscript. All the authors kindly acknowledge partial support by the RARE -318984 (an FP7 Marie Curie IRSES Fellowship). P. Liu acknowledges partial support by the Swiss National Science Foundation Project 200021-166274. C. Zhang also acknowledges partial support by the National Science Foundation of China 11371020.

\section{REFERENCES}

S. Asmussen and H. Albrecher. Ruin Probabilities. Second Edition. World Scientific, New Jersey, 2010.

J. Bertoin. Lévy processes, volume 121 of Cambridge Tracts in Mathematics. Cambridge University Press, Cambridge, 1996.

K. A. Borovkov and D. C. M. Dickson. On the ruin time distribution for a Sparre Andersen process with exponential claim sizes. Insurance Math. Econom., 42(3):1104-1108, 2008.

E. C. K. Cheung. Moments of discounted aggregate claim costs until ruin in a Sparre Andersen risk model with general interclaim times. Insurance Math. Econom., 53(2):343-354, 2013.

D. C. M. Dickson. The joint distribution of the time to ruin and the number of claims until ruin in the classical risk model. Insurance Math. Econom., 50(3):334-337, 2012.

F. Dufresne and H. U. Gerber. Risk theory for the compound Poisson process that is perturbed by diffusion. Insurance Math. Econom., 10(1):51-59, 1991.

A. D. Egídio dos Reis. How many claims does it take to get ruined and recovered? Insurance Math. Econom., $31(2): 235-248,2002$.

E. Frostig, S. M. Pitts, and K. Politis. The time to ruin and the number of claims until ruin for phase-type claims. Insurance Math. Econom., 51(1):19-25, 2012.

H. U. Gerber. An extension of the renewal equation and its application in the collective theory of risk. Skand. Aktuarietidskr., pages 205-210, 1970.

H. U. Gerber and E. S. W. Shiu. The joint distribution of the time of ruin, the surplus immediately before ruin, and the deficit at ruin. Insurance Math. Econom., 21(2):129-137, 1997.

H. U. Gerber and E. S. W. Shiu. On the time value of ruin. N. Am. Actuar. J., 2(1):48-78, 1998.

I. Karatzas and S. E. Shreve. Brownian motion and stochastic calculus, volume 113 of Graduate Texts in Mathematics. Springer-Verlag, New York, 1988. 
D. Landriault and T. Shi. First passage time for compound Poisson processes with diffusion: ruin theoretical and financial applications. Scand. Actuar. J., 4:368-382, 2014.

D. Landriault, T. Shi, and G. E. Willmot. Joint densities involving the time to ruin in the Sparre Andersen risk model under exponential assumptions. Insurance Math. Econom., 49(3):371-379, 2011.

J. M. L. Lee. The probability function of the number of claims until ruin in some risk models. Honours Research Essay. The University of Melbourne, 2011.

S. Li. The distribution of the dividend payments in the compound Poisson risk model perturbed by diffusion. Scand. Actuar. J., (2):73-85, 2006.

S. Li, F. Huang, and C. Jin. Joint distributions of some ruin related quantities in the compound binomial risk model. Stochastic Models, 29(4):518-539, 2013.

S. Li, Y. Lu, and C. Jin. Number of jumps in two-sided first-exit problems for a compound Poisson process. Methodol Comput Appl Probab., 18(3):747-764, 2016.

K. Sato. Lévy processes and infinitely divisible distributions, volume 68 of Cambridge Studies in Advanced Mathematics. Cambridge University Press, Cambridge, 1999. Translated from the 1990 Japanese original, Revised by the author.

C. C. L. Tsai and Y. Lu. An effective method for constructing bounds for ruin probabilities for the surplus process perturbed by diffusion. Scand. Actuar. J., (3):200-220, 2010.

C. Zhang and G. Wang. The joint density function of three characteristics on jump-diffusion risk process. Insurance Math. Econom., 32(3):445-455, 2003.

C. Zhao and C. Zhang. Joint density of the number of claims until ruin and the time to ruin in the delayed renewal risk model with Erlang(n) claims. J. Comput. Appl. Math., 244:102-114, 2013.

Peng Liu, Mathematical Institute, University of Wroceaw, Pl. Grunwaldzki 2/4, 50-384 Wroceaw, Poland, and Department of Actuarial Science, University of Lausanne, Unil-Dorigny, 1015 Lausanne, Switzerland

E-mail address: liupnankaimath@163.com

Chunsheng Zhang, School of Mathematical Sciences and LPMC, Nankai University, Tianjin 300071, China

E-mail address: zhangcs@nankai.edu.cn

Lanpeng Ji, Department of Actuarial Science, University of Lausanne, Unil-Dorigny, 1015 Lausanne, Switzerland, and Institute for Information and Communication Technologies, Heig-VD, University of Applied Sciences of Western Switzerland, Switzerland

E-mail address: jilanpeng@126.com 